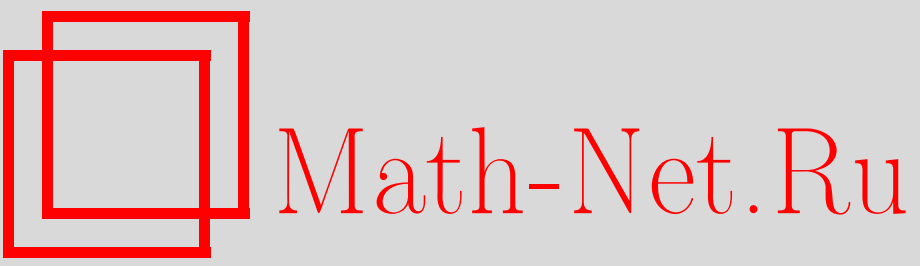

А. В. Калинкин, Точные решения уравнений Колмогорова для критических ветвящихся процессов с двумя комплексами взаимодействия частиц, УМH, 2001, том 56, выпуск $3,173-174$

DOI: https://doi.org/10.4213/rm408

Использование Общероссийского математического портала Math-Net.Ru подразумевает, что вы прочитали и согласны с пользовательским соглашением

http: //www . mathnet.ru/rus/agreement

Параметры загрузки:

IP : 3.80 .181 .102

26 апреля 2023 г., 12:03:57 


\title{
ТОЧНЫЕ РЕШЕНИЯ УРАВНЕНИЙ КОЛМОГОРОВА ДЛЯ КРИТИЧЕСКИХ ВЕТВЯЩИХСЯ ПРОЦЕССОВ С ДВУМЯ КОМПЛЕКСАМИ ВЗАИМОДЕЙСТВИЯ ЧАСТИЦ
}

\author{
А. В. КАЛИНКИн
}

Рассматривается однородный во времени марковский процесс $\xi_{t}$ со счетным множеством состояний $\{0,1,2, \ldots\}$ и непрерывным временем $t, t \in[0, \infty)$. Обозначим переходные вероятности $P_{i j}(t)=\mathrm{P}\left\{\xi_{t}=j \mid \xi_{0}=i\right\}, i, j=0,1, \ldots$, и пусть при $t \rightarrow 0+$ переходные вероятности имеют вид $(\lambda \geqslant 0, \mu \geqslant 0)$

$$
P_{i j}(t)= \begin{cases}\left(p_{j-i+2}^{2} i(i-1) \lambda+p_{j-i+1}^{1} i \mu\right) t+o(t), & \text { если } j \geqslant i-2, j \neq i \\ 1-(i(i-1) \lambda+i \mu) t+o(t), & \text { если } j=i ; \\ o(t), & \text { если } j \leqslant i-2,\end{cases}
$$

где $\left\{p_{l}^{k} \geqslant 0, l=0,1, \ldots ; \sum_{l=0}^{\infty} p_{l}^{k}=1, p_{k}^{k}=0\right\}, k=1,2,-$ заданные распределения вероятностей. Вводя производящие функции:

$$
F_{i}(t ; s)=\sum_{j=0}^{\infty} P_{i j}(t) s^{j}, \quad i=0,1, \ldots ; \quad h_{k}(s)=\sum_{l=0}^{\infty} p_{l}^{k} s^{l}, \quad k=1,2 ; \quad|s| \leqslant 1,
$$

вторую (прямую) систему дифференциалшных уравнений Колмогорова для переходных вероятностей свертьваем к равносилному уравнению в частных производных второго порядка параболического типа [1]-[3]

$$
\frac{\partial F_{i}(t ; s)}{\partial t}=\lambda\left(h_{2}(s)-s^{2}\right) \frac{\partial^{2} F_{i}(t ; s)}{\partial s^{2}}+\mu\left(h_{1}(s)-s\right) \frac{\partial F_{i}(t ; s)}{\partial s}, \quad F_{i}(0 ; s)=s^{i} .
$$

Для марковского процесса $\xi_{t}$ состояние $i$ интерпретируется как наличие совокупности из $i$ частиц типа $T$ [1], [4]. Можно полагать, что через случайное время $\tau_{i}^{2}, \mathrm{P}\left\{\tau_{i}^{2} \leqslant t\right\}=$ $1-e^{-i(i-1) \lambda t}$, происходит взаимодействие частицы типа $T$ с частицей типа $T$. Эта пара частиц независимо от всех других частиц превращается в новую группу из $n$ частиц типа $T$ с распределением $\left\{p_{n}^{2}\right\}$; процесс переходит в состояние $i+n-2$. Кроме того, через случайное время $\tau_{i}^{1}, \mathrm{P}\left\{\tau_{i}^{1} \leqslant t\right\}=1-e^{-\mu i t}$, одна из частиц типа $T$ независимо от других частиц превращается в группу из $m$ частиц типа $T$ с распределением $\left\{p_{m}^{1}\right\}$; процесс переходит в состояние $i+m-1$. Предполагается, что случайные величины $\tau_{i}^{1}, \tau_{i}^{2}$ независимы; в состоянии $i$ процесс $\xi_{t}$ находится случайное время $\tau_{i}=\min \left(\tau_{i}^{1}, \tau_{i}^{2}\right)$.

Основными характеристиками ветвящихся процессов [5] являются среднее число частиц $A_{i}(t)=\mathrm{E}\left(\xi_{t} \mid \xi_{0}=i\right)=\left.\left(\partial F_{i}(t ; s) / \partial s\right)\right|_{s=1}$ и дисперсия $B_{i}(t)=\mathrm{D}\left(\xi_{t} \mid \xi_{0}=i\right)$. Вводим параметры критичности $a_{2}=\lambda\left(h_{2}^{\prime}(1)-2\right), a_{1}=\mu\left(h_{1}^{\prime}(1)-1\right)$; полагаем $b_{2}=\lambda\left(h_{2}^{\prime \prime}(1)-2\right)$, $b_{1}=\mu h_{1}^{\prime \prime}(1)$. Для доказательства следуюшей теоремы дифференцируем (1) по $s$ и решаем обькновенное дифференциальное уравнение для $A_{i}(t)$; аналогичные выгисления для $B_{i}(t)$.

Теорема 1. Для критического $\left(a_{2}=0\right)$ прочесса $A_{i}(t)=i e^{a_{1} t} u$

$$
B_{i}(t)=\left(i(i-1)+\frac{i b_{1}}{b_{2}+a_{1}}\right) e^{\left(b_{2}+2 a_{1}\right) t}+\left(i-\frac{i b_{1}}{b_{2}+a_{1}}\right) e^{a_{1} t}-i^{2} e^{2 a_{1} t}
$$

Точные решения уравнения (1) строились в [4] и других работах в связи с приложениями в физике высоких энергий. В [2] дано решение для случая $h_{2}(s)=p_{0}+p_{1} s, h_{1}(s)=1$ в виде ряда Фурье. Рассматриваемые ниже частные случаи уравнения (1) решены с помощью замены переменной вида, указанного в [6] для уравнения 5.2.9.31.

Теорема 2. Решение линейного уравнения (1) при $\lambda=\mu u h_{2}(s)=\frac{1}{2} s^{4}+\frac{1}{2}, h_{1}(s)=s^{3}$ имеет вид

$$
F_{i}(t ; s)=\frac{1}{\sqrt{2 \pi \lambda t}} \int_{-\infty}^{\infty} e^{-v^{2} /(2 \lambda t)} \varphi^{i}(v ; s) d v,
$$


где дробно-линейная по переменной $s$ функция $\varphi(v ; s)=\frac{\operatorname{sh} v+s \operatorname{ch} v}{\operatorname{ch} v+s \operatorname{sh} v}$ удовлетворяет свойству итераций

$$
\varphi\left(v_{1} ; \varphi\left(v_{2} ; s\right)\right)=\varphi\left(v_{1}+v_{2} ; s\right)
$$

и нелинейной системе уравнений [7]:

$$
\begin{aligned}
& \left(h_{2}(s)-s^{2}\right)\left(\frac{\partial \varphi}{\partial s}\right)^{2}-\left(h_{2}(\varphi)-\varphi^{2}\right)=0 \\
& \left(h_{2}(s)-s^{2}\right) \frac{\partial^{2} \varphi}{\partial s^{2}}+\left(h_{1}(s)-s\right) \frac{\partial \varphi}{\partial s}-\left(h_{1}(\varphi)-\varphi\right)=0 .
\end{aligned}
$$

Для доказательства теоремы подставляем (2) в (1), функцию $\varphi(v ; s)$ в (3) и (4).

Ветвящийся процесс $\xi_{t}$ может выродиться в поглощающем состоянии 0. Для рассмотренного в теореме 2 случая из формулы (2) следует интегральное представление для вероятности невырождения процесса

$$
Q_{i}(t)=\mathrm{P}\left\{\xi_{t}>0 \mid \xi_{0}=i\right\}=1-F_{i}(t ; 0)=\frac{1}{\sqrt{2 \pi \lambda t}} \int_{-\infty}^{\infty} e^{-v^{2} /(2 \lambda t)}\left(1-\operatorname{th}^{i} v\right) d v ;
$$

отсюда получаем $Q_{i}(t) \equiv 1$ для нечетных $i$, для четных $i$ - асимптотическую формулу при $t \rightarrow \infty$

$$
Q_{i}(t) \sim C_{i} \cdot \frac{1}{\sqrt{\lambda t}}, \quad C_{i}=\sqrt{\frac{2}{\pi}} \sum_{k=1}^{i / 2} \frac{1}{2 k-1} .
$$

Впервые выявленное свойство (3) для марковского процесса с взаимодействием аналогично свойству итераций производящей функции переходных вероятностей обычного (когда в уравнении (1) $\lambda=0$ ) ветвящегося процесса, являющимуся основным функциональным соотношением теории ветвящихся процессов [5; гл. $1, \S 4]$. Формулы $(2)$ и $(5)$ указьвают на связь процессов с взаимодействием и ветвящихся процессов в случайной среде [8]. Интеграл в выражении (2) интерпретируется как математическое ожидание [7] для дробно-линейной производящей функции распределения числа потомков [5; гл. $1, \S 8$, пример 2], [8], а асимптотическая формула (5) совпадает с полученной в [8] формулой для вероятности невырождения критического ветвящегося процесса в случайной среде.

Теорема 3. Решение уравнения (1) при $\lambda=\mu u h_{2}(s)=\frac{1}{2} s^{3}+\frac{1}{2} s, h_{1}(s)=\frac{3}{4} s^{2}+\frac{1}{4}$ имеет вид (2), где функция

$$
\varphi(v ; s)=\left(\frac{\operatorname{sh}(v / 2)+\sqrt{s} \operatorname{ch}(v / 2)}{\operatorname{ch}(v / 2)+\sqrt{s} \operatorname{sh}(v / 2)}\right)^{2}
$$

удовлетворяет свойству (3) и системе уравнений (4). При $t \rightarrow \infty$

$$
Q_{i}(t) \sim C_{i} \cdot \frac{1}{\sqrt{\lambda t}}, \quad C_{i}=\sqrt{\frac{8}{\pi}} \sum_{k=1}^{i} \frac{1}{2 k-1} .
$$

\section{СПИСОК ЛИТЕРАТУРЫ}

[1] Б. А. Севастьянов, А. В.Калинкин // Докл. АН СССР. 1982. Т. 264. № 2. C. 306-308. [2] A. Kalinkin, G. Valent // Обозрение прикл. промшшл. матем. Сер. вероятн. и статист. 1998. V. 5. №2 2. Р. 304-305. [3] А. В. Калинкин // Теория вероятн. и ее примен. 2001. T. 46. № 2. [4] J. Letessier, G. Valent // Approx. Theory Appl. 1988. V. 4. № 2. Р. 97-117. [5] Б. А. Севастьянов. Ветвящиеся процессы. М.: Наука, 1971. [6] В. Ф. Зайцев, А. Д. Полянин. Справочник по диффференциальным уравнениям с частными производными: Точные решения. М.: МПО, 1996. [7] А. В. Калинкин // Докл. РАН. 2000. Т. 371. № 2. С. 159-162. [8] М. В. Козлов // Теория вероятн. и ее примен. 1976. Т. 21. № 4. С. 813-825.

Московский государственньй технический университет им. Н.Э. Баумана 